\title{
Optical coherence tomography predicts visual outcome in macula-involving rhegmatogenous retinal detachment
}

This article was published in the following Dove Press journal:

Clinical Ophthalmology

10 January 2012

Number of times this article has been viewed

\author{
Minhee Cho',* \\ Matthew T Witmer ${ }^{1, *}$ \\ Guilleherme Favarone ${ }^{2}$ \\ RV Paul Chan' \\ Donald J D'Amico' \\ Szilárd Kiss' \\ 'Weill Cornell Medical College, \\ Department of Ophthalmology, \\ New York, NY, USA; ${ }^{2}$ Hospital do Olho \\ Rio Preto, Department of Retina and \\ Vitreous, São José do Rio Preto, Brazil \\ *These authors contributed equally \\ to this work
}

Correspondence: Szilárd Kiss

Weill Cornell Medical College,

Department of Ophthalmology,

I 305 York Ave, I Ith Floor,

New York, NY 10021 , USA

$\mathrm{Tel}+\mathrm{I} 6469622020$

Fax + I 6469620602

Emailszk700I@med.cornell.edu
Purpose: Visual recovery after rhegmatogenous retinal detachment (RRD) repair depends upon various anatomical factors. We investigated spectral-domain optical coherence tomography (SD-OCT) abnormalities, pre- and postoperatively, in patients with nontraumatic RRD and correlated these findings with visual outcome.

Methods: The medical records of all patients presenting to Weill Cornell Medical College with nontraumatic macula-involving RRD from August 2010 to September 2011 were retrospectively reviewed in this single-center, consecutive case series. All patients underwent pre- and postoperative visual acuity (VA) testing, slit-lamp biomicroscopy, and dilated fundus examination. Spectral domain optical coherence tomography was obtained preoperatively in twelve patients and postoperatively in ten patients.

Results: Twelve patients (12 eyes) were included in the final analysis. Preoperative optical coherence tomography revealed that the inner segment/outer segment (IS/OS) junction was disrupted in 10/12 eyes (83\%), the external limiting membrane (ELM) was disrupted in $9 / 12$ (75\%) eyes, cystoid macular edema (CME) was present in 10/12 (83\%) eyes, an epiretinal membrane (ERM) was present in 2/12 eyes (17\%) and outer retinal corrugation was present in 7/12 (58\%) eyes. In postoperative imaging of 10 eyes, the IS/OS junction was disrupted in 4/10 (40\%), the ELM was disrupted in 3/10 (30\%) eyes, CME was present in 2/10 (20\%), and an ERM in 1/10 (10\%). All retinas were attached postoperatively. Outer retinal corrugation was the most predictive of worse preoperative $(P=0.0016)$ and 1 -month postoperative visual acuity $(P=0.05)$.

Conclusion: Preoperative SD-OCT demonstrating outer retinal corrugation in macula involving RRD predicts poor visual acuity outcome in nontraumatic RRD. Such findings may have implications for the urgency for these eyes to undergo surgical repair.

Keywords: macula-involving, optical coherence tomography, outer retinal corrugation, retinal detachment

\section{Introduction}

Rhegmatogenous retinal detachment (RRD) leads to anatomical distortion of the human retina and consequently, visual impairment. Despite excellent surgical success rates and anatomical success with the current modalities of treatment, visual outcomes may still be disappointing. Important predictors of visual recovery after retinal detachment surgery include preoperative visual acuity (VA) and the presence of foveal detachment. ${ }^{1-4}$ Decreased VA after successful repair of retinal detachment may be due to epiretinal membrane, cystoid macular edema, ${ }^{5,6}$ retinal folds, ${ }^{7}$ and persistent foveal detachment., ${ }^{8,9}$

Optical coherence tomography (OCT) remains an important diagnostic and monitoring tool for all macular diseases and is able to image retinal anatomy essentially 
to the level of clinical histology. Several studies have shown an association between either the preoperative or postoperative structural changes detected on OCT with postoperative visual outcome after RRD repair. ${ }^{10-13}$

In this study, we investigated spectral-domain (SD) OCT abnormalities, pre- and postoperatively, in patients with macula involving RRD and correlated these findings with visual outcome.

\section{Materials and methods}

This study was a retrospective, nonrandomized, singlecenter, consecutive case series. The medical records of patients who underwent surgical repair of a macula-involving nontraumatic RRD at Weill Cornell Eye Associates between August 2010 and September 2011 were reviewed. The macula was defined as the area of retina located between the temporal vascular arcades, approximately $6 \mathrm{~mm}$ in diameter, centered at the fovea. The fovea was defined as the central retinal depression, approximately $4.0 \mathrm{~mm}$ temporal and $0.8 \mathrm{~mm}$ inferior to the center of the optic disc, and was approximately $1.5 \mathrm{~mm}$ in diameter. The Institutional Review Board approved the study. Only those patients with SD-OCT (Spectralis HRA+OCT; Heidelberg Engineering, Inc, Heidelberg, Germany) in the preoperative evaluation were included. When possible, patients also received SD-OCT imaging postoperatively.

All patients underwent pre- and postoperative VA testing, slit-lamp biomicroscopy, and dilated fundus examination. Other data collected included demographics, duration of symptoms, presence of foveal involvement, time to surgery, type of surgical repair, and complications. Patients with other retinal diseases that could affect the OCT results such as diabetic retinopathy, retinal vein occlusions, and chorioretinal dystrophies were excluded. OCT characteristics were analyzed qualitatively. OCT characteristics were described and correlated with final visual outcome. Snellen VA was converted to $\log$ MAR (log of the minimum angle of resolution) for statistical analysis. The following conversion to logarithm of the minimum angle of resolution was used for vision worse than 20/400: counting fingers, 1.6; hand motion, 2.0; light perception, 2.5; and no light perception, 3.0. Statistical analysis consisted of unpaired $t$-tests to compare means using GraphPad Prism software (Graphpad Software Inc, La Jolla, CA).

\section{Results}

The patient demographics and preoperative OCT findings are displayed in Table 1. Twelve eyes of 12 patients aged
Table I Preoperative: OCT findings

\begin{tabular}{llll}
\hline Total patients & 12 & & \\
Macula-involving & 12 & \\
Fovea-involving & 10 & \\
Male & 6 & \\
Mean age (range, years) & 62.7 & $(25-70)$ \\
Right eye & 5 & \\
Days from OCT to surgery (range) & 2.2 & $(0-10)$ \\
Preoperatively & & & \\
Mean visual acuity (logMAR) & 0.9 & \\
Mean duration of symptoms (days) & 11 & $(1-30)$ \\
OCT characteristics present & & Mean VA & \\
IS/OS preserved & 2 & 0.69 \\
IS/OS disrupted & 10 & 1.12 \\
ELM preserved & 3 & \\
ELM disrupted & 9 & \\
CME & 10 & \\
ERM & 2 & \\
ORC present & 7 & 1.24 \\
ORC absent & 5 & 0.42 \\
Surgery & & & \\
PPV & 12 & &
\end{tabular}

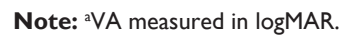

Abbreviations: CME, cystoid macular edema; IS/OS, inner segment/outer segment junction; ELM, external limiting membrane; ERM, epiretinal membrane; ORC, outer retinal corrugation; PPV, pars plana vitrectomy; VA, visual acuity.

25 to 70 years were included (mean age, 62.7 years). Preoperatively, patients had an average of 11 days of symptoms (range, 1 to 30 days) prior to presentation, which included flashing lights, floaters, a black curtain, or blurred vision. The time to surgical repair from the day of presentation ranged from 0 to 10 days (mean, 2.2 days). Preoperative VA ranged from 20/30 to finger counting with mean $\log$ MAR of 0.9 .

The inner segment/outer segment junction (IS/OS) of the outer retina was preserved in only two of 12 eyes preoperatively (Figures 1 and 2). The external limiting membrane (ELM) was preserved in three eyes and disrupted in nine. Ten of 12 eyes

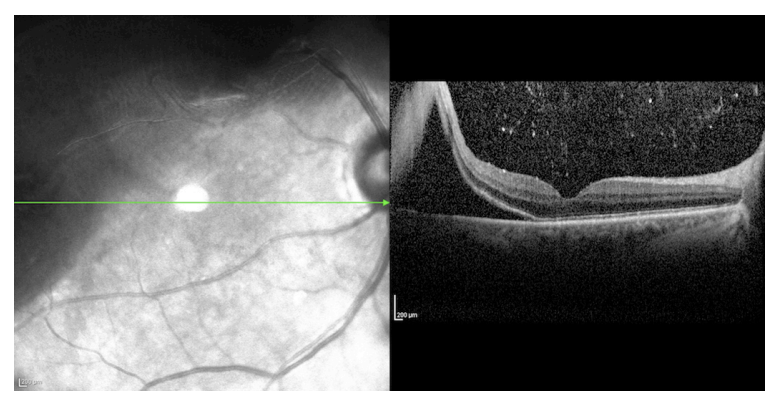

Figure I Preoperative optical coherence tomography of the right eye of a 69-year-old female with a rhegmatogenous retinal detachment. The macula is detached, but the fovea is attached. Preoperative visual acuity (VA) was 20/30. The external limiting membrane and inner segment/outer segment junction of the photoreceptors are preserved under the fovea. There is no evidence of outer retinal corrugation. There is an absence of cystoid macular edema. The detachment was surgically repaired with a pars plana vitrectomy. The VA I-month postoperatively was $20 / 30$. 


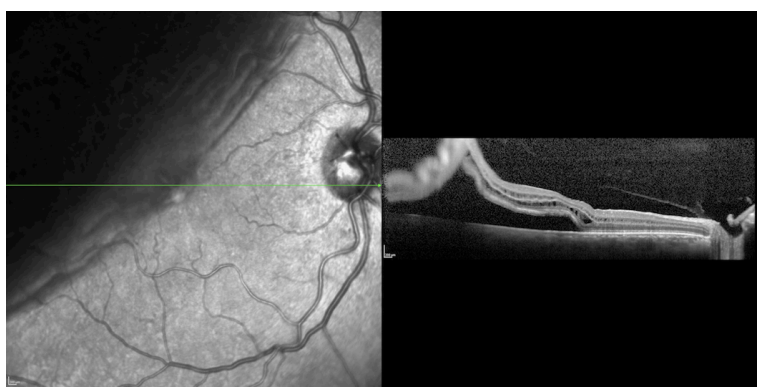

Figure 2 Preoperative optical coherence tomography (OCT) of the right eye of a 53-year-old female with a rhegmatogenous retinal detachment. The macula and the fovea are detached. Preoperative visual acuity (VA) was 20/40. The external limiting membrane (ELM) was preserved, but the inner segment/outer segment (IS/OS) junction of the photoreceptors was disrupted. There is an absence of outer retinal corrugation, but presence of cystoid macular edema. The detachment was surgically repaired with a pars plana vitrectomy. The postoperative OCT revealed a preserved ELM and IS/OS junction. The VA I-month postoperatively remained 20/40.

had cystoid macular edema (CME) and two eyes demonstrated epiretinal membranes (ERM). Seven eyes had outer retinal corrugation (ORC) (Figure 3). The preoperative VA of eyes with ORC was a mean of 1.24 , while the mean VA of those without ORC was $0.42(P=0.0016)$.

All twelve eyes underwent surgical repair via pars plana vitrectomy. The retina of each eye included in the study was attached with a single surgery and remained attached at the last follow-up visit.

Ten patients underwent SD-OCT postoperatively and their OCT characteristics are displayed in Table 2. Postoperative best-corrected VA ranged from $20 / 30$ to $20 / 250$ with a mean of $\operatorname{logMAR} 0.52$. The OCT was performed an average 37.7 days (range, $21-83$ days) after the surgery. The IS/OS junction was preserved in three of ten eyes, intact but segmented in three of ten (Figure 4), and severely disrupted in the remaining (4/10). The ELM was preserved in seven eyes and disrupted in the remaining. Two eyes had CME and one eye

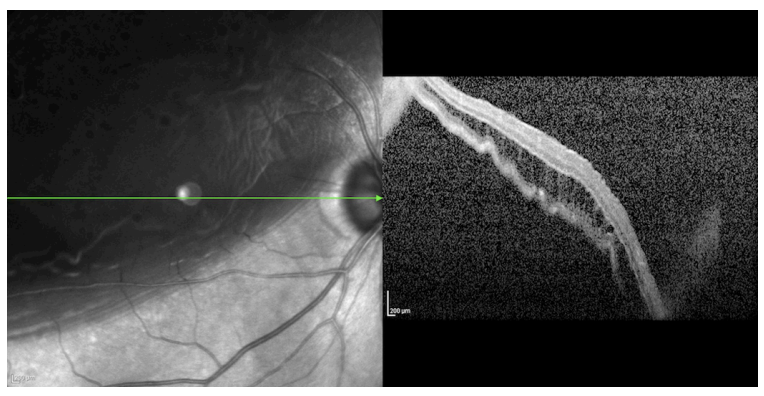

Figure 3 Preoperative optical coherence tomography (OCT) of the right eye of a 67-year-old female with a rhegmatogenous retinal detachment. The macula and the fovea are detached. Preoperative visual acuity (VA) was 20/400. The external limiting membrane and the inner segment/outer segment (IS/OS) junction of the photoreceptors were disrupted. There is evidence of outer retinal corrugation and cystoid macular edema (CME). The detachment was surgically repaired with a pars plana vitrectomy. The postoperative ELM and IS/OS junction remained disrupted on OCT. The VA I-month postoperatively was 20/60.
Table 2 Postoperative OCT results

\begin{tabular}{lllll}
\hline Eyes imaged with OCT & 10 & & \\
postoperatively & & & \\
Mean VA (logMAR) & 0.52 & & \\
Mean days from surgery & 37.7 & $(2 I-83)$ & & \\
to OCT (range, days) & & & \\
Fovea-involving RD & 8 & & \\
Fovea-sparing RD & 2 & & \\
& & Mean VAa & \\
IS/OS preserved or segmented & 6 & 0.32 & \\
IS/OS disrupted & 4 & 0.74 & \\
ELM preserved & 7 & & \\
ELM disrupted & 3 & & \\
CME & 2 & & \\
ERM & 1 & & \\
SRF & 1 & & \\
ORC present postoperatively & 0 & & \\
& & Mean PO VA & \\
ORC present preoperatively & 6 & 0.66 & \\
ORC absent preoperatively & 4 & 0.24 & \\
\hline Not & & &
\end{tabular}

Note: ${ }^{2} V A$ measured in logMAR.

Abbreviations: CME, cystoid macular edema; IS/OS, inner segment/outer segment junction; ELM, external limiting membrane; ERM, epiretinal membrane; ORC, outer retinal corrugation; PO, postoperative; PPV, pars plana vitrectomy; SRF, subretinal fluid; VA, visual acuity.

had an ERM. The postoperative VA of eyes with preoperative ORC was a mean of 0.66 , while the postoperative mean VA of eyes without preoperative ORC was $0.24(P=0.05)$. The mean postoperative VA of eyes with disrupted IS/OS junction on OCT postoperatively was 0.74 , while the mean VA of eyes with a postoperative preserved or segmented IS/OS junction was $0.32(P=0.20)$.

The pre- and postoperative OCT findings of each patient are displayed in Table 3.

\section{Discussion}

Optical coherence tomography has been used to investigate the pathology of retinal detachment for over a decade. ${ }^{14}$

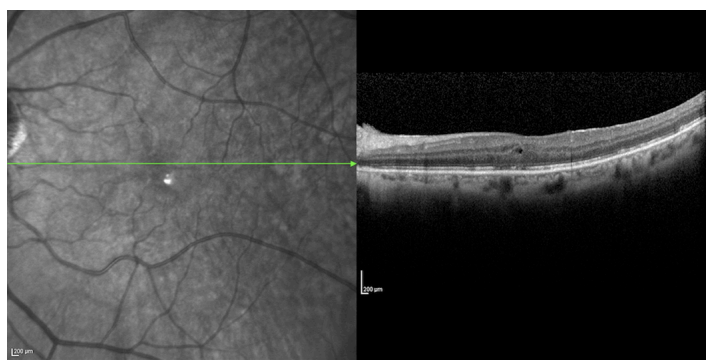

Figure 4 Postoperative optical coherence tomography (OCT) of the left eye of a 59-year-old female who had presented with a rhegmatogenous retinal detachment, which was repaired with a pars plana vitrectomy. The macula and the fovea were detached preoperatively. Preoperative visual acuity (VA) was 20/200. The external limiting membrane (ELM) and inner segment/outer segment (IS/OS) junction of the photoreceptors were disrupted. There was presence of outer retinal corrugation and cystoid macular edema. This postoperative OCT reveals persistent CME with a preserved ELM layer, but segmented IS/OS junction. The VA I-month postoperatively was $20 / 60$. 
Table 3 Individual patient data

\begin{tabular}{|c|c|c|c|c|c|c|c|c|c|c|c|c|c|c|}
\hline & \multirow[t]{2}{*}{ Age } & \multirow[t]{2}{*}{ Sex } & \multirow[t]{2}{*}{ Eye } & \multirow[t]{2}{*}{ Fovea $^{a}$} & \multicolumn{5}{|c|}{ Preoperative VA and OCT findings } & \multicolumn{5}{|c|}{ Postoperative VA and OCT findings ${ }^{b}$} \\
\hline & & & & & VA & IS/OS & ELM & CME & ORC & VA & IS/OS & ELM & CME & ORC \\
\hline I & 69 & $\mathrm{~F}$ & OD & ON & 0.18 & Preserved & Preserved & Absent & No & 0.18 & Preserved & Preserved & Absent & No \\
\hline 2 & 61 & $\mathrm{~F}$ & OS & ON & 0.48 & Preserved & Preserved & Absent & No & 0.30 & Preserved & Preserved & Absent & No \\
\hline 3 & 47 & M & OD & OFF & 1.60 & Disrupted & Disrupted & CME & Yes & 1.10 & Disrupted & Disrupted & CME & No \\
\hline 4 & 67 & $M$ & OD & OFF & 1.30 & Disrupted & Disrupted & CME & Yes & 0.48 & Disrupted & Disrupted & Absent & No \\
\hline 5 & 56 & $M$ & OD & OFF & 0.30 & Disrupted & Disrupted & CME & Yes & 0.30 & Disrupted & Preserved & Absent & No \\
\hline 6 & 98 & $M$ & OS & OFF & 1.30 & Disrupted & Disrupted & CME & Yes & 1.10 & Disrupted & Disrupted & Absent & No \\
\hline 7 & 53 & $\mathrm{~F}$ & OD & OFF & 0.30 & Disrupted & Preserved & CME & No & 0.30 & Preserved & Preserved & Absent & No \\
\hline 8 & 59 & $\mathrm{~F}$ & OS & OFF & 1.00 & Disrupted & Disrupted & CME & Yes & 0.48 & Segmented & Preserved & CME & No \\
\hline 9 & 50 & $\mathrm{~F}$ & OS & OFF & 0.60 & Disrupted & Disrupted & CME & No & 0.18 & Segmented & Preserved & Absent & No \\
\hline 10 & 64 & $M$ & OS & OFF & 1.60 & Disrupted & Disrupted & CME & Yes & 0.48 & Segmented & Preserved & Absent & No \\
\hline 11 & 65 & $M$ & OS & OFF & 1.60 & Disrupted & Disrupted & CME & Yes & $\mathrm{N} / \mathrm{A}$ & $\mathrm{N} / \mathrm{A}$ & N/A & $\mathrm{N} / \mathrm{A}$ & $\mathrm{N} / \mathrm{A}$ \\
\hline 12 & 63 & $\mathrm{~F}$ & OS & OFF & 0.54 & Disrupted & Disrupted & CME & No & $\mathrm{N} / \mathrm{A}$ & N/A & $N / A$ & $N / A$ & $\mathrm{~N} / \mathrm{A}$ \\
\hline
\end{tabular}

Notes: aPreoperative foveal attachment status by OCT; bone-month postoperatively.

Abbreviations: CME, cystoid macular edema; ELM, external limiting membrane; IS/OS, inner segment/outer segment junction line; ORC, outer retinal corrugation; $N / A$, not available; VA, visual acuity measured in logMAR.

The initial report by Hagimura and colleagues evaluated 25 eyes with macula involving retinal detachment and found that $60 \%$ of eyes demonstrated intraretinal separation, and $30 \%$ of eyes demonstrated an undulated outer retina. ${ }^{14}$ Intraretinal separation, intraretinal separation with an undulated outer retina, and height of the detachment at the foveal center were all associated with poor best-corrected VA. ${ }^{14}$ Eyes with normal retinal architecture had significantly better best-corrected VA. The duration of time from the onset of symptoms to the OCT was not associated with the presence of intraretinal separation and intraretinal separation occurred as soon as 3 days after the onset of subjective symptoms in some patients. ${ }^{14}$ This particular report, however, only evaluated eyes preoperatively. ${ }^{14}$

Optical coherence tomography has also been used in the past to demonstrate the behavior of the retina after retinal detachment surgery. The reports by Wolfensberger and colleagues and Hagimura and colleagues used OCT to demonstrate delayed attachment of the fovea in eyes treated with scleral buckling procedures. ${ }^{8,9}$ In the study by Hagimura and colleagues, despite the fact that all 15 eyes $(100 \%)$ appeared attached by ophthalmoscopy at 1 month, $47 \%$ of eyes demonstrated foveal detachment 1 month postoperatively using OCT. ${ }^{8}$ The final VA substantially increased when the attachment of the fovea occurred and the authors of both reports suggested that delayed visual recovery after retinal reattachment with scleral buckling surgery may be due to persistent foveal detachment. ${ }^{8,9}$ The report by Wolfensberger and colleagues used OCT to demonstrate that the retina was reattached much more consistently and quickly with vitrectomy than with scleral buckling in macula-affecting detachments. ${ }^{15}$ At 1 month postoperation,
$67 \%$ of eyes treated with scleral buckle demonstrated subfoveal fluid while $0 \%$ of eyes treated with vitrectomy had subfoveal fluid..$^{15}$

Several reports have analyzed the structural changes found on OCT of the macula in patients with macula-involving retinal detachments. ${ }^{10,13}$ Lecleire-Collet and colleagues analyzed RRD using time-domain OCT (Stratus OCT; Carl Zeiss Meditec AG, Dublin, CA) and observed preoperative cystic cavities in the inner and outer nuclear layers, height of the detachment at the fovea, duration of detachment, and postoperative lack of return of the junctional line between the IS and OS of the photoreceptors after 9 months were correlated with poorer final VA. ${ }^{10}$ The postoperative integrity of the IS/OS junction as a predictor of VA was also subsequently shown in another report. ${ }^{12}$ It is important to mention here that recently, the use of the term "inner segment/outer segment" junction line to refer to the second distinct line in the outer retina as displayed by SD-OCT has been challenged, and that perhaps the more anatomically accurate term for this line should be the "ellipsoid layer."16 For historical consistency, and because this controversy remains unresolved, we have chosen to continue to refer to this layer of the OCT as the IS/OS junction in this report. Time-domain OCT also has been used to correlate the thickness of the outer nuclear layer and the height of the retinal detachment with worse postoperative VA. ${ }^{17}$

Few studies have reported SD-OCT findings in patients with RRD. ${ }^{11,13}$ Nakanishi and colleagues observed cystoid cavities in the inner nuclear layer, cystoid macular edema in the outer nuclear layer, undulations of the photoreceptor layer, and dropout of IS/OS of photoreceptor layers in the macula. ${ }^{13}$ In that study, the dropout of foveal photoreceptor 
IS/OS (which occurred in $40 \%$ of eyes), was correlated with preoperative and postoperative VA. ${ }^{13}$ In addition, height of the retinal detachment was inversely correlated with preand postoperative VA. ${ }^{13}$ Pre- or postoperative VA was not correlated with duration of symptoms. ${ }^{13}$ Wakabayashi and colleagues ${ }^{11}$ studied the postoperative status of ELM and IS/OS junction with ultrahigh-resolution (UHR) OCT in RRD patients and concluded that, at 6 months of follow-up, the rate of IS/OS junction restoration differed significantly in the eyes with or without intact ELM on the initial postoperative UHR-OCT. The IS/OS junction was restored in $64 \%$ of eyes with an intact ELM postoperatively, while it was restored in $0 \%$ of eyes without an intact ELM postoperatively. ${ }^{11}$ In addition, the postoperative VA was directly correlated with the integrity of the ELM and IS/OS junction layers, with eyes with both layers intact averaging 20/19 VA and eyes with both layers disrupted averaging 20/80. ${ }^{11}$ The ability of the ELM and IS/OS junction to become restored months after $\mathrm{RD}$ repair was also subsequently demonstrated by Lai and colleagues. ${ }^{12}$ The authors concluded that it is possible that the integrity of ELM is important in determining the reversibility of photoreceptor damage after an RRD. ${ }^{11}$

As demonstrated in the current study, high-resolution imaging afforded by SD-OCT allows for interpretation of retinal anatomy to histologic precision and enables detection of retinal abnormalities in eyes with RRD. In our study, we show that the presence of ORC on preoperative OCT is predictive of worse preoperative VA and early postoperative visual outcome. The presence of this sign upon OCT portends a worse visual prognosis for patients with maculainvolving RRDs.

There are several potential reasons why eyes with ORC have a worse visual prognosis. First, this finding may indicate more severe photoreceptor damage at the time of detachment, which may be irreversible. It is also possible that this sign is a manifestation of chronicity for patients with $\mathrm{RD}$. In our series, however, patients with ORC preoperatively presented with a shorter mean duration of symptoms than patients without ORC on preoperative OCT (10 days versus 16 days). Alternatively, the presence of ORC may be related to the mechanism of detachment (ie, location and extent of retinal break) or the retinal anatomy of the patient. Clearly, however, further study is required to determine the true reason for the development of ORC.

The limitations of this study include the small sample size, the retrospective design of the study, and the subjective nature of the interpretations of the images. The strength of this paper is the inclusion of both pre- and postoperative images.
ORC was strengthened as a risk factor for poor visual outcome after successful RRD repair. Currently, this particular risk factor can only be detected using OCT technology. As with most findings on OCT, this risk factor is best defined with newer SD-OCT technology compared to time-domain OCT. The imaging technology investigated in this study is a valuable tool that not only contributes to our understanding of the pathophysiology of RRD, but also aids clinicians in the surgical planning and counseling and may provide patients with an appropriate expectation for visual recovery after surgery.

\section{Acknowledgment}

This project was made possible in part by a generous unrestricted grant to the Weill Cornell Department of Ophthalmology from the Research to Prevent Blindness.

\section{Disclosure}

No authors have proprietary or conflict of interest in any of the materials present in this manuscript.

\section{References}

1. Sharma T, Challa JK, Ravishankar KV, et al. Scleral buckling for retinal detachment. Predictors for anatomic failure. Retina. 1994;14(4): 338-343.

2. Grizzard WS, Hilton GF, Hammer ME, et al. A multivariate analysis of anatomic success of retinal detachments treated with scleral buckling. Graefes Arch Clin Exp Ophthalmol. 1994;232(1):1-7.

3. Wilkinson CP. Visual results following scleral buckling for retinal detachments sparing the macula. Retina. 1981;1(2):113-116.

4. Ross WH, Stockl FA. Visual recovery after retinal detachment. Curr Opin Ophthalmol. 2000;11(3):191-194.

5. Sabates NR, Sabates FN, Sabates R, et al. Macular changes after retinal detachment surgery. Am J Ophthalmol. 1989;108(1):22-29.

6. Heimann H KB. Primary vitrectomy in rhegmatogenous retinal detachment. In: Ryan S, Hinton D, Schachat A, et al, editors. Retina. 4th ed. Philadelphia, PA: Elsevier Mosby; 2006:2085-2094.

7. Tani P, Robertson DM, Langworthy A. Prognosis for central vision and anatomic reattachment in rhegmatogenous retinal detachment with macula detached. Am J Ophthalmol. 1981;92(5):611-620.

8. Hagimura N, Iida T, Suto K, et al. Persistent foveal retinal detachment after successful rhegmatogenous retinal detachment surgery. Am J Ophthalmol. 2002;133(4):516-520.

9. Wolfensberger TJ, Gonvers M. Optical coherence tomography in the evaluation of incomplete visual acuity recovery after macula-off retinal detachments. Graefes Arch Clin Exp Ophthalmol. 2002;240(2): 85-89.

10. Lecleire-Collet A, Muraine M, Menard JF, et al. Evaluation of macular changes before and after successful retinal detachment surgery using stratus-optical coherence tomography. Am J Ophthalmol. 2006;142(1): 176-179.

11. Wakabayashi T, Oshima Y, Fujimoto H, et al. Foveal microstructure and visual acuity after retinal detachment repair: Imaging analysis by Fourier-domain optical coherence tomography. Ophthalmology. 2009; 116(3):519-528.

12. Lai WW, Leung GY, Chan CW, et al. Simultaneous spectral domain OCT and fundus autofluorescence imaging of the macula and microperimetric correspondence after successful repair of rhegmatogenous retinal detachment. Br J Ophthalmol. 2010;94(3):311-318. 
13. Nakanishi H, Hangai M, Unoki N, et al. Spectral-domain optical coherence tomography imaging of the detached macula in rhegmatogenous retinal detachment. Retina. 2009;29(2):232-242.

14. Hagimura N, Suto K, Iida T, et al. Optical coherence tomography of the neurosensory retina in rhegmatogenous retinal detachment. Am J Ophthalmol. 2000;129(2):186-190.

15. Wolfensberger TJ. Foveal reattachment after macula-off retinal detachment occurs faster after vitrectomy than after buckle surgery. Ophthalmology. 2004;111(7):1340-1343.
16. Spaide RF, Curcio CA. Anatomical correlates to the bands seen in the outer retina by optical coherence tomography: Literature review and model. Retina. 2011;31(8):1609-1619.

17. Maruko I, Iida T, Sekiryu T, et al. Morphologic changes in the outer layer of detached retina in rhegmatogenous retinal detachment and central serous chorioretinopathy. Am J Ophthalmol. 2009;147(3):489-494.

\section{Publish your work in this journal}

Clinical Ophthalmology is an international, peer-reviewed journal covering all subspecialties within ophthalmology. Key topics include: Optometry; Visual science; Pharmacology and drug therapy in eye diseases; Basic Sciences; Primary and Secondary eye care; Patient Safety and Quality of Care Improvements. This journal is indexed on

\footnotetext{
Submit your manuscript here: http://www.dovepress.com/clinical-ophthalmology-journal
}

\section{Dovepress}

PubMed Central and CAS, and is the official journal of The Society of Clinical Ophthalmology (SCO). The manuscript management system is completely online and includes a very quick and fair peer-review system, which is all easy to use. Visit http://www.dovepress.com/ testimonials.php to read real quotes from published authors. 